TABLE OF CONTENTS

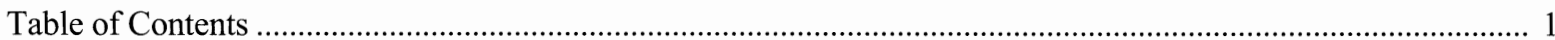

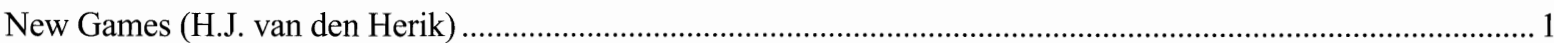

The Quad Heuristic in Lines of Action (M.H.M. Winands, J.W.H.M. Uiterwijk, and H.J van den Herik) .............. 3

Synthesis of Chess and Chess-like Endgames: A Proof of Correctness (K. P. Coplan) ...................................... 16

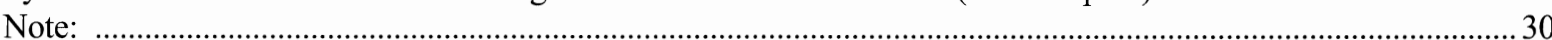

Review:

Multigame - an Environment for Distributed Game Tree Research (D. Hartmann)..............................35

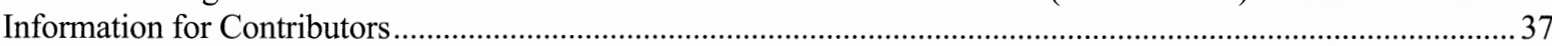

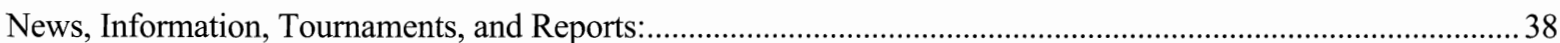

The Match Van der Wiel vs. ReBel CenTury 3.0 (J. van Reek and J.W.H.M. Uiterwijk) ................. 38

The $10^{\text {th }}$ International Paderborn Computer-Chess Championship (U. Lorenz)................................. 42

The First Open Mediocrity Programming Competition (H. Iida and M. Hlynka) ............................ 46

The Second International Conference on Computers and Games (J. van Rijswijck and M. Müller).... 49

The Sixth Computer Olympiad (Maastricht, 2001) ..........................................................................5

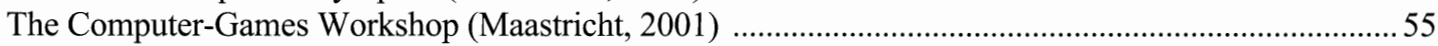

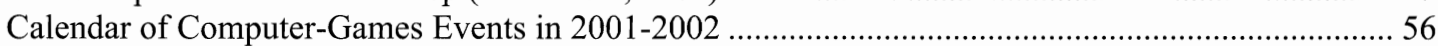

Obituaries:

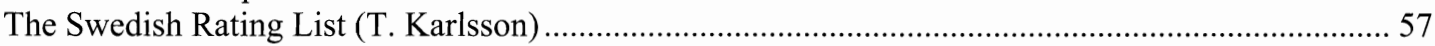

Claude E. Shannon (1916-2001) (K. Thompson; H.J. van den Herik).............................................. 58

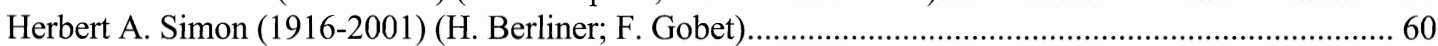

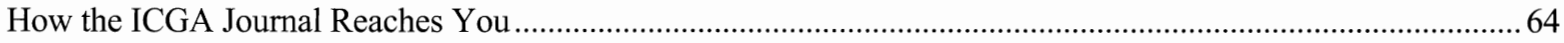

\title{
NEW GAMES
}

Do we really need new games? This question quickly emerges when a discussion is initiated on the fundamentals of the International Computer Games Association Journal, the ICGA Journal. In 1999 the ICCA Journal left the preserve of chess to broaden its scope and to increase interest in game-playing programs in general. Many game-playing specialists with some affection towards computers had in mind games close to chess, such as checkers and Go, and their more distant relatives Chinese Chess and Shogi. They turned out to be wrong, since there are many more games around, as could be read in the lists of the participating programs in the five Computer Olympiads held so far. There we have seen a total of 23 games. Four of them disappeared, having been solved (Connect-Four, Qubic, Go-Moku, and Nine Men's Morris) and this issue reports on a claim that a fifth Olympic game has also been solved, viz. Renju.

However, a game can be solved without knowing the exact strategy; then it is said that the game has been solved ultra-weakly. For instance, the game of Hex is a first-player win as proven by John Nash, although so far the winning strategy is unknown. To complicate matters a swap rule has been introduced implying that after the first move the second player may choose which side to play. As a consequence of this rule the first player will always choose a move which does not look too good. For Hex, this is difficult since in that game it holds that the first move is win preserving or losing (no draws are possible). So, a need for strategic concepts to understand the power of a move remains on our research list.

From the games mentioned above it seems that two issues are important: (1) solving a game and (2) developing a winning strategy. In the past we have seen scientific progress in both areas. When looking in the restricted domain of endgames, especially chess endgames, we notice that our scientific vocabulary has been enriched by various metrics, such as distance to conversion, distance to mate, distance to zeroing move, and depth by the rule, as well as by an attempt to introduce a measure of beauty.

Yet, the original aim of computer-chess (computer-checkers) researchers was to obtain insight into the human brain processes when grandmasters were playing chess or checkers. Of course, a second goal was to develop a program of World-Champion calibre. Accordingly, the emphasis of these researchers was on developing techniques that should eventually result in strong playing programs. 
The growth of computer power - or otherwise stated, the effect of Moore's law - is that in the future more games will be solved, or will be endowed with such powerful techniques that they will play at a level not understandable to human beings. Is this the end of computer-games research? In principle, it is, if no new games are invented, developed, or found in ancient history. Thus any respected Journal on games should look for new games, since 'to stand still is to move back'.

Therefore we are pleased to introduce the readers into the intricacies of a new game called Mediocrity. In February 2001 the First Open Mediocrity Programming Competition was held in Hamamatsu, Japan. Mediocrity is played by $n$ players and for this competition $n$ was set at 3 . To stimulate programming games the Information Processing Society of Japan (IPSJ) always organizes a side event during its annual Programming Symposium, called Games and Puzzles Competitions on Computers (GPCC). In 2000, it was decided that Mediocrity was one of the new games at the GPCC 2001. The rules, the tournament, and information on the participating programs are reported in this issue. Moreover, another new game, called Ackanomic, is referred to.

Playing games is fun and that must be the point of departure, but some games are brain teasing and then a computer can help. This is true for old games, such as Domineering or Dots and Boxes, as well as for the new ones mentioned above. Fortunately, the world of games is rather diverse. So, besides chess-like games, we have connection games, mancala games, racing games, games of chance, strategy games, and negotiating games.

All in all it is astonishing how many games there are and it is fascinating that due to the current opportunities of computing and communication they are less of a mystery. Dakon has been solved by a human brain, but the proof was given by a computer. Many configurations of Kalah, including the official one have been solved (see ICGA Journal, Vol. 23, No. 3); more solutions of Tchuka Ruma are expected to be next in line. Tchuka Ruma is a traditional game from Malaysia and the Philippines. It is played on a circular board with a ring of holes in it. One of the holes is called the 'Ruma'; it is larger than the other ones. At the start, all holes except the Ruma are filled with an equal number of stones. The goal of the game is to collect as many stones in the Ruma as possible. It is a one-player game and the rules for sowing are Awari-like. Tchuka Ruma can be played with a different number of holes and stones. The game can best be compared to Solitaire and Rubik's Cube. So far the game has been solved for configurations with $\leq 10$ pits and up to 100,000 stones per pit. This is but one example of the many games in which mathematics and logic perform side by side. Other challenging games are Gipf, Tamsk, Zèrtz, Twixt, and Onyx.

The exposition above almost indicates that the new century is starting with new ideas and new games. This might be the case, but with much sadness we would also like to pay our sincere respect to two giants of our games world: Claude Shannon and Herb Simon. Both passed away in the first quarter of 2001; both had an enormous impact on science in general. For them the world of chess was only an application domain for their theories. Shannon was a front-ranked researcher in Boolean circuitry, the entropy of information, the cryptography and Artificial Intelligence. For Herb Simon the research domains were complementary, i.e., economy, rational behaviour, cognitive science, the physical-symbol system, and Artificial Intelligence. We are grateful for the contributions they made to our domain as can be read in the Obituaries.

Finally, we return to the question: do we need new games? The answer is clearly affirmative. Only by analysing, programming, and attempting to solve new games are we able to develop new techniques and consequently to understand better the intricacies of the games, the secrets of computer technology, and the enormous range of applications for the techniques developed in our game park.

Jaap van den Herik

ICGA Journal readers may be interested to know that information on our publications is available on the Internet. Our homepage can be reached by http://www.dcs.qmw.ac.uk/ icca/journal.htm

The e-mail address of the ICCA Maastricht has been changed into CS-ICCA-L@cs.unimaas.nl.

A Japanese homepage can be reached by http://www.cs.inf.shizuoka.ac.jp/ iida/icga/

A complete list of all articles, notes, and literature reviews published in the ICCA Journal and the ICGA Journal is available on the Internet at http://www.des.qmw.ac.uk/ icca/toc.htm 